Case Report

\title{
Mycobacterium haemophilum as the Initial Presentation of a B-Cell Lymphoma in a Liver Transplant Patient
}

\author{
T. Doherty, M. Lynn, A. Cavazza, E. Sames, and R. Hughes \\ Ashford and St. Peters NHS Trust, Chertsey, Surrey KT16 OPZ, UK \\ Correspondence should be addressed to T. Doherty; iamteresadoherty@gmail.com \\ Received 6 November 2013; Accepted 26 November 2013; Published 12 January 2014 \\ Academic Editors: G. S. Alarcon, S. Koarada, G. O. Littlejohn, and M. Salazar-Paramo
}

Copyright (C) 2014 T. Doherty et al. This is an open access article distributed under the Creative Commons Attribution License, which permits unrestricted use, distribution, and reproduction in any medium, provided the original work is properly cited.

\begin{abstract}
A 66-year-old woman presented with pustular lesions of her face, trunk, and limbs and an acute arthritis of the knees and elbows. She had a complex medical background and had been on immunosuppressants for three years after a liver transplant. Tissue samples from her skin lesions and synovial fluid showed acid-fast bacilli. Mycobacterium haemophilum, an atypical mycobacteria, was later grown on culture. During her treatment with combination antibiotic therapy, she developed a pronounced generalised lymphadenopathy. Histology showed features of a diffuse B-cell lymphoma, a posttransplant lymphoproliferative disorder (PTLD).
\end{abstract}

\section{Presentation}

In October 2012 a 66-year-old, Filipino, retired psychiatric nurse attended the rheumatology department in a wheelchair. She gave a 3-week history of painful and swollen knees, ankles, and elbows. Walking had become difficult. Systemically she was unwell and described weight loss (five kilograms over a month), malaise, and anorexia. Widespread tender and raised pustular skin lesions (Figures 1 and 2) were noted over her face, arms, chest, and legs. She reported that these were itchy and painful and had failed to improve with a two-week course of oral flucloxacillin (500 mg QDS). Further examination revealed that she was clinically anaemic and confirmed a large joint polyarthritis with bilateral knee effusions. Other systems examination was unremarkable.

\section{Past Medical History and Medications}

Her significant medical history included a liver transplant, in 2009, for a primary malignant neoplasm (hepatocellular carcinoma).

Her other past medical history included idiopathic thrombocytopenic purpura, type 2 diabetes (diet controlled), chronic kidney disease (stage 3), vitamin B12 deficiency, a hysterectomy (for menorrhagia), and secondary osteoporosis due to steroids.

As part of her antirejection regime she had been taking mycophenolate mofetil (2 grams daily) and low dose prednisolone (5 milligrams daily).

She also took alendronic acid ( $70 \mathrm{mg}$ weekly) and calcium carbonate/cholecalciferol (1.5 g/400 IU daily). For her recent joint pains she had been using paracetamol and codeine.

\section{Investigations}

Initial blood tests showed a $\mathrm{Hb}$ of $7.0 \mathrm{~g} / \mathrm{dL}$ (hypochromic and microcytic), CRP $168 \mathrm{mg} / \mathrm{L}$, WCC $9.6 \times 10^{9} / \mathrm{L}$, neutrophils 9.0 $\times 10^{9} / \mathrm{L}$, urea $10 \mathrm{mmol} / \mathrm{L}$, and creatinine $122 \mu \mathrm{mol} / \mathrm{L}$.

She was admitted to hospital and transfused with three units of blood.

Investigations included an upper gastrointestinal endoscopy which revealed multiple shallow ulcers and positive Helicobacter pylori urease test. Her left knee aspirate showed scanty positively birefringent crystals on microscopy and pseudomonas on Gram staining. A mid-stream urine sample grew E. coli. Electrocardiograms and a chest X-ray were normal. CT of her chest, abdomen, and pelvis was reported as normal aside from a left upper kidney pole abnormality, 


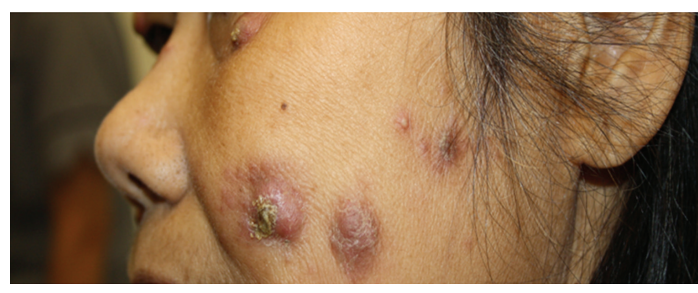

FIGURE 1

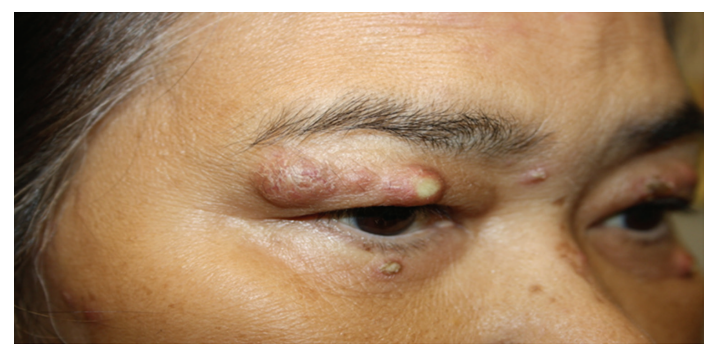

FIGURE 2

which was likely to represent a cyst. This was later reviewed by the urologists who were unconcerned but arranged followup.

\section{Initial Management and Further Microbiology Results}

For her septic arthritis and her skin infection she was commenced on intravenous meropenem (1 gram TDS). She received eradication treatment for the Helicobacter (one week of twice-daily omeprazole $20 \mathrm{mg}$, metronidazole $400 \mathrm{mg}$, and clarithromycin $250 \mathrm{mg}$ ). Further microbiology results from a skin lesion biopsy and a knee synovial aspirate both showed Pseudomonas bacilli and stained strongly positive for acidfast bacilli (AFB). Samples of synovial fluid from the knee were sent to the National Tuberculosis Reference Laboratory in London for further characterization. This showed that the AFB were Mycobacterium tuberculosis (MTB) complex negative indicating an atypical mycobacterium. Culture results followed showing a rare atypical AFB, M. haemophilum.

She was initiated on the recommended regime of a combination of oral ciprofloxacin (500 mg BD), clarithromycin (500 $\mathrm{mg} \mathrm{BD})$, and rifampicin (300 $\mathrm{mg} \mathrm{BD})$ and discharged home. On this combination she had an initial, nonsustained response (of one month) in terms of her skin lesions and joints, but she relapsed requiring rehospitalisation. She was then commenced on a combination of oral clarithromycin $500 \mathrm{mg}$ BD and intravenous imipenem QDS which led to clinical improvements. She was discharged home with the community team administering her antibiotics.

\section{Complications}

Over the subsequent months she was reviewed on a monthly basis in the rheumatology outpatients department. At her review in February 2013, she had a palpable right preauricular $(1 \mathrm{~cm})$ and a left groin lymph node $(1.5 \mathrm{~cm})$.
A repeat CT of her chest, abdomen, and pelvis showed new subcutaneous lymphadenopathy in the anterior abdominal wall and in the flanks at the level of her fifth lumbar vertebra. An enlarging node was noted in the left ischiorectal fossa and there were two abnormal nodes in the right hemipelvis.

Histology of the left groin node showed lymph node tissue containing medium-to-large sized lymphocytes with a few inflammatory cells and small spotty foci of necrosis with a few neutrophil polymorphs in keeping with a high grade lymphoma.

Immunohistochemistry was positively staining for CD20, CD79a, bcl-2, CD30 (focal), and bcl-6 (focal). There was lambda restriction, $\mathrm{T}$ cell markers showed reactive $\mathrm{T}$ cells, and MIB-1 was increased (60\% expression), all consistent with a diffuse large B-cell lymphoma.

She went on to have a positron electron tomography (PET) scan which also correlated with a diagnosis of lymphoma.

She was promptly seen by the haematologists who made the decision to treat her lymphoma with rituximab. We maintained regular outpatient followup and continued her antibiotics for her skin lesions which appeared to worsen with any attempt to withdraw from the regime.

Eleven months on from her initial presentation she is clinically well. She has residual skin lesions (eighty percent improvement) and she has completed rituximab treatment. A reduction in her mycophenolate to $500 \mathrm{mg} \mathrm{BD}$ was sanctioned by her liver specialist and we intend to complete twelve months of her current antibiotics before stopping (10 months completed).

\section{Discussion}

Human M. haemophilum infection was first diagnosed by Sompolinsky in 1978. This first description of the infection manifested as cutaneous lesions in a 51-year-old female with Hodgkin's lymphoma [1].

Since this report, there have been over 220 published cases of human $M$. haemophilum infecting mainly immunecompromised adults and healthy children. The most recent review was done by Lindeboom et al. in 2011 [2]. The adult infections most frequently involve the skin, but there are a number of case reports with septic arthritis [3], disseminated [4] and pulmonary infections [5], pyomyositis [6], osteomyelitis [7], and ophthalmic involvement [8, 9]. Rarely, the involvement of other systems has been reported. There has been one case report of an epididymal abscess in a patient with a renal transplant [10] and central nervous system involvement in two patients [11].

Immunocompetent adults have been known to acquire cutaneous infections transmitted in permanent makeup or tattoo ink [12-14]. In children, cervicofacial lymphadenitis is the most common manifestation and is not associated with immunocompromise [15].

In veterinary medicine $M$. haemophilum has been isolated in zebra fish and useful epidemiology studies have been conducted in reservoirs. Studies have concluded that identical 
mycobacterium strains have been found infecting fish and in the biofilms, but more research is needed [16]. In 2002 Hernandez-Divers et al. published a case of a royal python with pulmonary mycobacteriosis due to dual infections of $M$. haemophilum and M. marinum [17]. Pai et al. in 2003 isolated M. haemophilum from hospital cockroaches in China and speculated on their role in transmitting the infection [18]. In 2006 Jacob et al. reported a case of a M. haemophilum epidural granuloma in an American bison [19].

Despite the case reports, it is an infection that may be underdiagnosed. The reasons are its unusual culture requirements of an iron enriched (ferric ammonium citrate or hemin) medium and the low incubation temperatures of 30 to $32^{\circ} \mathrm{C}$ for growth in vitro. In their review in 1996, Saubolle et al. recommended requesting such cultures in immunocompromised patients with septic arthritis or skin lesions, bone marrow transplant patients with pulmonary lesions, adenitis in children and in situations where acid-fast strains from previous specimens were acid-fast positive, but the cultures were negative [20].

The natural habitat and the mode of transmission remain unclear. There has been speculation that contaminated water sources have borne infection in humans and fish, although no clinical isolates have been directly linked to environmental isolates. The virulence is considered low, mainly based on animal studies where healthy mice and guinea pigs were inoculated with large volumes of bacilli (intravenously, subcutaneously and intramuscularly) and suffered no ill effects. However mice who were given prednisolone developed ear lesions with AFBs similar to cutaneous lesions seen in human adults [21].

The treatment can be complex and prolonged and removing the cause of immunosuppression is advocated [22]. In Lindeboom et al's review they described 33 cutaneous infections with $M$. haemophilum in immunocompromised adults [2]. Antibiotic regimens usually included clarithromycin and were most commonly inclusive of ciprofloxacin and rifampicin. Other antibiotics used were doxycycline, ethambutol, rifabutin, isoniazid, cycloserine, amikacin, azithromycin, trimethoprim-sulfamethoxazole, pyrazinamide, minocycline, gatifloxacin, and levofloxacin. The duration of the antibiotic treatments varied from one to forty-two months. None of the regimens for cutaneous lesions included imipenem, but amongst twelve patients with disseminated disease, one cardiac transplant patient with skin, pulmonary and joint involvement was successfully treated with a combination of imipenem, clarithromycin, ciprofloxacin, and doxycycline.

This lady's diagnosis was delayed and she failed to sustain a response to the most commonly accepted regimens and those antibiotic recommended by the National Tuberculosis Centre. Unfortunately there is no evidence based approach for an alternative regime, but we continued to rely on the antibiotic regimen which appeared to have generated a sustained response. Therefore we continued intravenous imipenem (QDS) and per oral clarithromycin (BD).

This lady had several risk factors for the development of $M$. haemophilum including her background of a liver transplant and subsequent immunosuppression with prednisolone and mycophenolate. Her vulnerability was enhanced by her diabetes mellitus and the underlying undiagnosed B-cell lymphoma. She continues to make significant improvements on the protracted course of antibiotics, the treatment of her lymphoma, and the reduction of her mycophenolate dose.

The responses to antibiotics are not uniform, but several authors have published reports of success with prolonged combination therapy. Seeking advice from the National Tuberculosis Referencing Centres is recommended, but therapy should be measured against clinical responses.

This is the first case report of M. haemophilum preceeding a diagnosis of lymphoma. Our case is recognisably complex requiring multiple diagnostic steps with specialist input from rheumatology, dermatology, microbiology, haematology, hepatology, and community healthcare teams.

A number of case reports of Mycobacterium haemophilum in patients with an underlying rheumatological diagnosis have also been published. The first case report of a patient with a diagnosis of lupus treated with mycophenolate before developing the infection was published in 2002 [23].

Patients on biologic treatments for inflammatory arthritis have also developed $M$. haemophilum infections [24]. We support Saubolle et al's recommendation for iron enriched low temperature cultures of synovial fluids or skin biopsies in immunocompromised patients. This case has served to increase our awareness of this infection in patients presenting to the rheumatology with acute arthritides and those patients under rheumatology followup on immunosuppressants.

\section{Conflict of Interests}

The authors declare that there is no conflict of interests regarding the publication of this paper.

\section{References}

[1] D. Sompolinsky, A. Lagziel, D. Naveh, and T. Yankilevitz, "Mycobacterium haemophilum sp. nov., a new pathogen of humans," International Journal of Systematic Bacteriology, vol. 28, no. 1, pp. 67-75, 1978.

[2] J. A. Lindeboom, L. E. S. B. van Coppenraet, D. van Soolingen, J. M. Prins, and E. J. Kuijper, "Clinical manifestations, diagnosis, and treatment of Mycobacterium haemophilum infections," Clinical Microbiology Reviews, vol. 24, no. 4, pp. 701-717, 2011.

[3] R. J. Olsen, P. L. Cernoch, and G. A. Land, "Mycobacterial synovitis caused by slow-growing nonchromogenic species: eighteen cases and a review of the literature," Archives of Pathology and Laboratory Medicine, vol. 130, no. 6, pp. 783-791, 2006.

[4] C. F. Kelley, W. S. Armstrong, and M. E. Eaton, "Disseminated Mycobacterium haemophilum infection," The Lancet Infectious Diseases, vol. 11, no. 7, pp. 571-578, 2011.

[5] D. A. White, T. E. Kiehn, A. Y. Bondoc, and S. A. Massarella, "Pulmonary nodule due to Mycobacterium haemophilum in an immunocompetent host," American Journal of Respiratory and Critical Care Medicine, vol. 160, no. 4, pp. 1366-1368, 1999.

[6] E.-Y. Jang, S.-O. Lee, S.-H. Choi et al., "Case of pyomyositis due to Mycobacterium haemophilum in a renal transplant recipient," Journal of Clinical Microbiology, vol. 45, no. 11, pp. 3847-3849, 2007. 
[7] S. Elsayed and R. Read, "Mycobacterium haemophilum osteomyelitis: case report and review of the literature," $B M C$ Infectious Diseases, vol. 6, article 70, 2006.

[8] M. J. Millar, C. Bulliard, C. Balachandran, and A. J. Maloof, "Mycobacterium hemophilum infection presenting as filamentary keratopathy in an immunocompromised adult," Cornea, vol. 26, no. 6, pp. 764-766, 2007.

[9] D. Modi, D. Pyatetsky, D. P. Edward et al., "Mycobacterium haemophilum a rare cause of endophthalmitis," Retina, vol. 27, no. 8, pp. 1148-1151, 2007.

[10] M. Keller, A. Mak, L. Thibert, P. Rene, and M. B. Klein, "Mycobacterium haemophilum epididymal abscess in a renal transplant patient," Journal of Clinical Microbiology, vol. 46, no. 7, pp. 2459-2460, 2008.

[11] P. Phowthongkum, A. Puengchitprapai, N. Udomsantisook, S. Tumwasorn, and C. Suankratay, "Spindle cell pseudotumor of the brain associated with Mycobacterium haemophilum and Mycobacterium simiae mixed infection in a patient with AIDS: the first case report," International Journal of Infectious Diseases, vol. 12, no. 4, pp. 421-424, 2008.

[12] M. K. Kay, T. R. Perti, and J. S. Duchin, "Tattoo-associated Mycobacterium haemophilum skin infection in immunocompetent adult, 2009," Emerging Infectious Diseases, vol. 17, no. 9, pp. 1734-1736, 2011.

[13] C. Hamsch, W. Hartschuh, A. Enk, and K. Flux, "A chinese tattoo paint as a vector of atypical mycobacteria-outbreak in 7 patients in Germany," Acta Dermato-Venereologica, vol. 91, no. 1, pp. 63-64, 2011.

[14] U. Wollina, "Nodular skin reactions in eyebrow permanent makeup: two case reports and an infection by Mycobacterium haemophilum," Journal of Cosmetic Dermatology, vol. 10, no. 3, pp. 235-239, 2011.

[15] J. A. H. Lindeboom, C. F. Kuijper, and M. Van Furth, "Inguinal lymphadenitis caused by Mycobacterium haemophilum in an immunocompetent child," Pediatric Infectious Disease Journal, vol. 26, no. 1, pp. 84-86, 2007.

[16] C. M. Whipps, C. Lieggi, and R. Wagner, "Mycobacteriosis in zebrafish colonies," ILAR Journal, vol. 53, pp. 95-105, 2012.

[17] S. J. Hernandez-Divers and D. Shearer, "Pulmonary mycobacteriosis caused by Mycobacterium haemophilum and M marinum in a royal python," Journal of the American Veterinary Medical Association, vol. 220, no. 11, pp. 1661-1663, 2002.

[18] H.-H. Pai, W. C. Chen, and C. F. Peng, "Isolation of nontuberculous mycobacteria from hospital cockroaches (Periplaneta americana)," Journal of Hospital Infection, vol. 53, no. 3, pp. 224-228, 2003.

[19] B. Jacob, B. M. DeBey, and D. Bradway, "Spinal intradural Mycobacterium haemophilum granuloma in an American Bison (Bison bison)," Veterinary Pathology, vol. 43, no. 6, pp. 998-1000, 2006.

[20] M. A. Saubolle, T. E. Kiehn, M. H. White, M. F. Rudinsky, and D. Armstrong, "Mycobacterium haemophilum: microbiology and expanding clinical and geographic spectra of disease in humans," Clinical Microbiology Reviews, vol. 9, no. 4, pp. 435447, 1996.

[21] M. R. Abbott and D. D. Smith, "The pathogenic effects of Mycobacterium haemophilum in immunosuppressed albino mice," Journal of Medical Microbiology, vol. 13, no. 4, pp. 535540, 1980.

[22] J. A. Lindeboom, "Surgical treatment for non tuberculous mycobacterium (NTM) cervicofacial lymhadenitis in children,"
Journal of Oral and Maxillofacial Surgery, vol. 70, no. 2, pp. 345348, 2012.

[23] C. L. Teh, K. O. Kong, A. P. Y. Chong, and H. Badsha, "Mycobacterium haemophilum infection in an SLE patient on mycophenolate mofetil," Lupus, vol. 11, no. 4, pp. 249-252, 2002.

[24] A. Aslam, R. L. Green, L. Motta, M. Ghrew, C. E. Griffiths, and R. B. Warren, "Cutaneous Mycobacterium haemophilum infection in a patient receiving infliximab for psoriasis," British Journal of Dermatology, vol. 168, no. 2, pp. 446-447, 2013. 


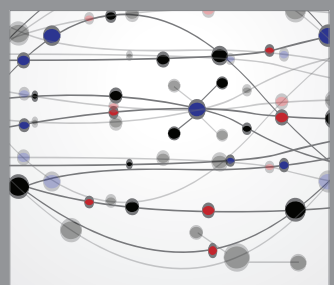

The Scientific World Journal
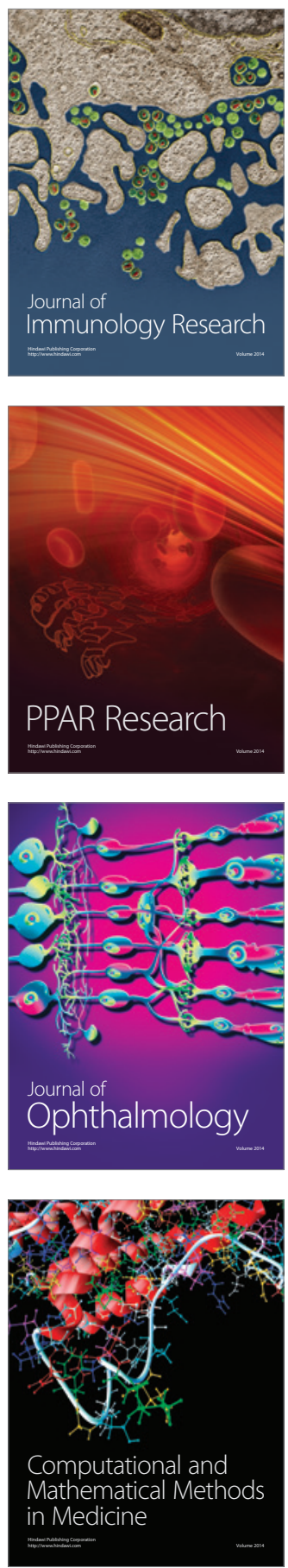

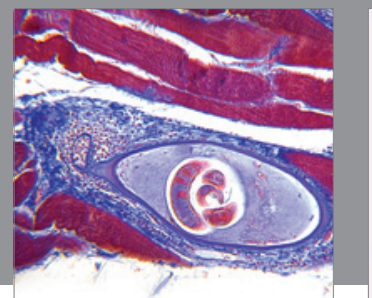

Gastroenterology

Research and Practice
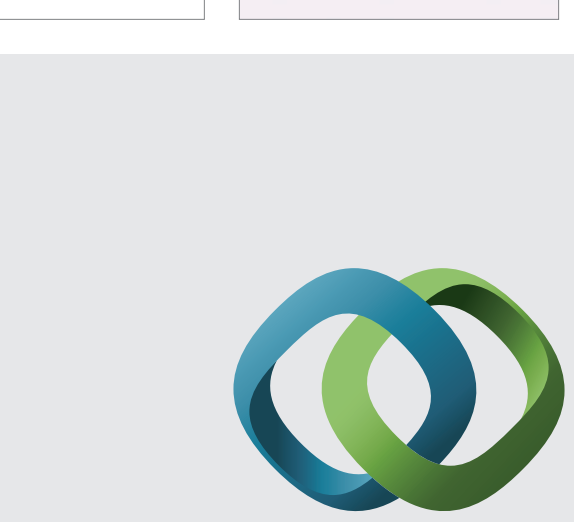

\section{Hindawi}

Submit your manuscripts at

http://www.hindawi.com
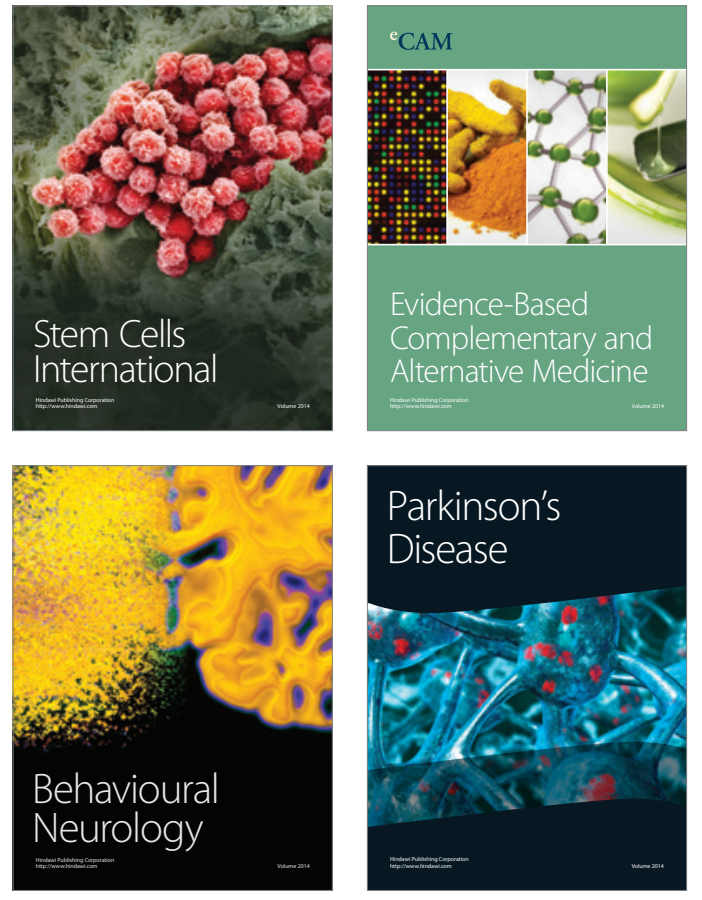
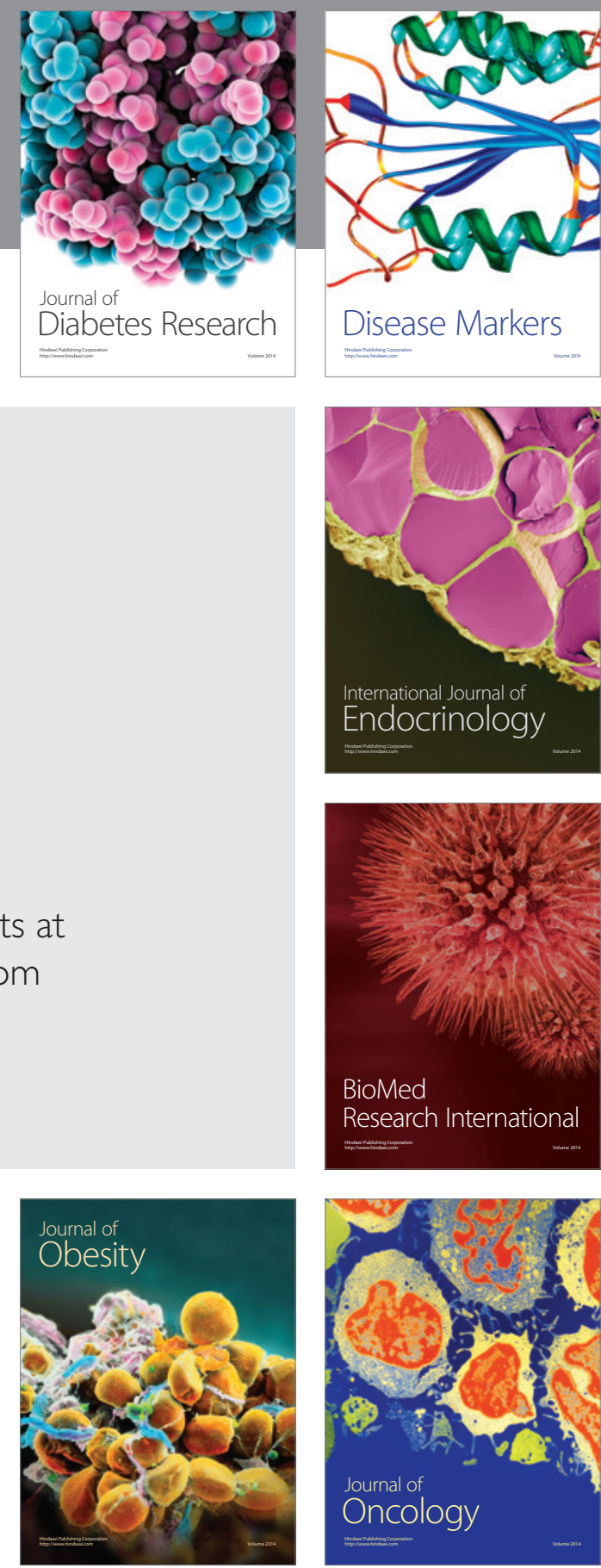

Disease Markers
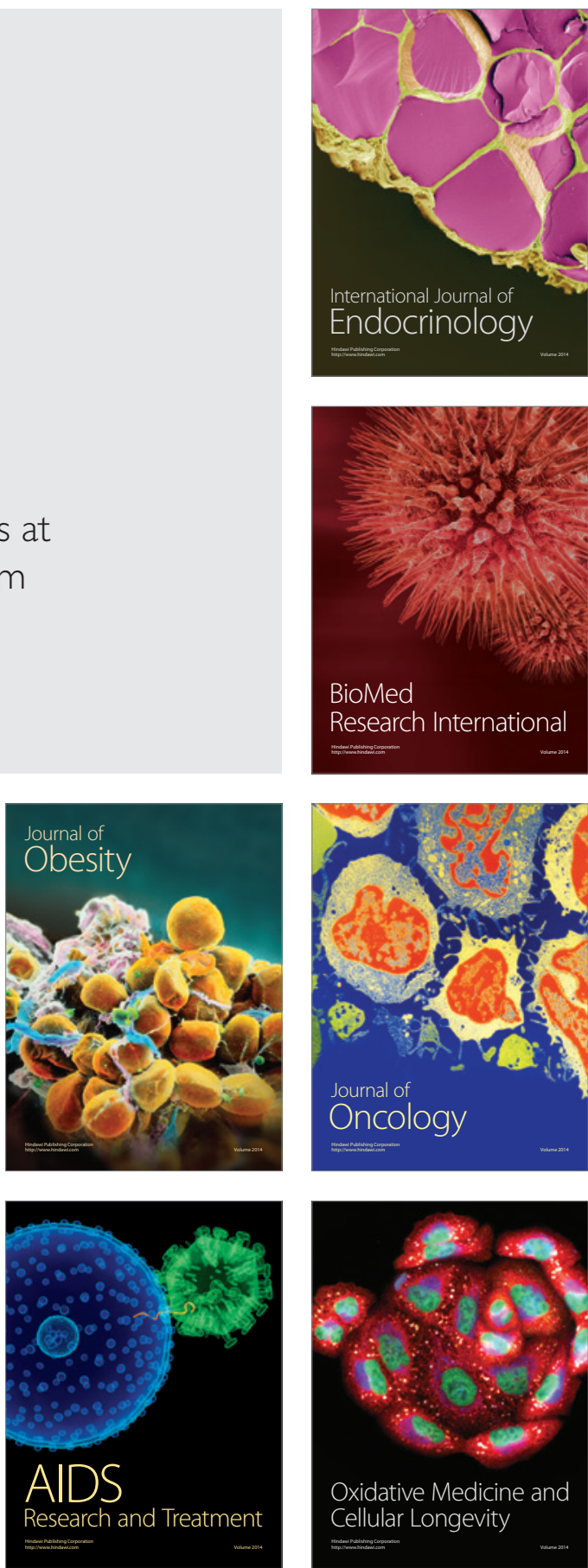